Rev. Fav. Agron. (LUZ). 2007, 24 Supl. 1: 422-426

\title{
Fitobentos y macrófitas de la cuenca del río Manzanares, estado Sucre, Venezuela
}

\section{(Phytobenthos and macrophytes of the Manzanares river basin, Sucre state, Venezuela)}

\author{
J. Barrios ${ }^{1}$, S. Sinatra y W. Senior
}

${ }^{1}$ Departamento de Biología Marina. Instituto Oceanográfico de Venezuela. Universidad de Oriente.

\section{Resumen}

Se realizó un inventario del fitobentos y macrófitas de la cuenca del río Manzanares, estado Sucre, Venezuela, entre noviembre de 2003 y julio de 2005. La colecta se hizo manualmente y el material se examinó con una lupa estereoscópica y microscopio. Con ayuda de claves artificiales se identificaron un total de 21 especies de fitobentos y macrófitas: 4 Cyanophyta, 9 Chlorophyta, 1 Heterokontophyta, 2 Rhodophyta, 3 Bryophyta y 2 Magnoliophyta. La cuenca baja del Manzanares presentó el mayor número de especies, siendo las más ampliamente distribuidas las Chlorophyta Spirogyra majuscula y Cladophora fracta y la Bryophyta Fontinalis squamosa. La contaminación del río Manzanares favorece el incremento de algas verdes filamentosas.

Palabras clave: Inventario, fitobentos, macrófitas, contaminación.

\section{Abstract}

A phytobenthos and macrophytes checklist was carried at the Manzanares river basin, Sucre state, Venezuela, from November 2003 to July 2005. Collect was accomplished manually and material was examined with a stereoscopic loupe and a microscope. With artificial key aid, 21 phytobenthos and macrophytes species: 4 Cyanophyta, 9 Chlorophyta, 1 Heterokontophyta, 2 Rhodophyta, 3 Bryophyta and 2 Magnoliophyta were identified. The basin lowers of the Manzanares presented the higher species number, being the Chlorophyta Spirogyra majuscula and Cladophora fracta and the Bryophyta Fontinalis squamosa the most extensively distributed. The Manzanares river pollution favors the filamentous green algae development.

Key words: Checklist, phytobenthos, macrophytes, pollution.

$\overline{\text { Recibido el 9-1-2007 }}$ - Aceptado el 30-4-2007

Autor para correspondencia e-mail: jebar@sucre.udo.edu.ve 


\section{Introducción}

Los organismos de agua dulce comprenden una gran cantidad de grupos taxonómicos, representando el fitobentos y las macrófitas en conjunto un significativo componente de la biodiversidad de estos ambientes, jugando un papel importante como productores primarios y en la dinámica estructural de ríos, lagos, represas, etc (1). Las algas, musgos, pteridofitas y fanerógamas son comunes en los cuerpos de agua dulce de Venezuela, no obstante, este conjunto de organismos ha sido poco estudiado. En este trabajo se realizó un listado del fitobentos y macrófitas del río Manzanares, Estado Sucre, Venezuela, como parte de un estudio de línea base para conocer la biodiversidad en la cuenca de este río.

La cuenca del río Manzanares forma parte del sistema montañoso del Turimiquire (Estado Sucre) y constituye una fuente de agua potable de gran importancia para la región oriental, ubicándose entre las coordenadas $10^{\circ} 05^{\prime} 30^{\prime \prime}-10^{\circ} 29^{\prime} 20^{\prime \prime} \mathrm{LN}$ y entre $63^{\circ} 45^{\prime} 30^{\prime \prime}-63^{\circ} 19^{\prime} 20^{\prime \prime}$ LO. El río Manzanares desemboca en el Golfo de Cariaco, formando una pluma de descarga laminar que se extiende por toda la zona costera de la ciudad de Cumaná. Para este trabajo se seleccionaron 24 estaciones a lo largo del Manzanares y sus tributarios.

\section{Materiales y métodos}

Las muestras se colectaron entre noviembre de 2003 y julio de 2005 . Se colectaron manualmente las macrófitas y fitobentos fijados a cualquier tipo de substrato y se refrigeraron en cavas con hielo hasta su traslado al laboratorio, en donde se preservaron con formalina al $5 \%$ y se almacenaron en frascos de boca ancha con sus respectivos datos. El estudio de los ejemplares completos se efectuó con una lupa estereóscopica, y se procesaron cortes histológicos realizados a mano alzada con una hojilla de acero según la técnica modificada de Womersley (4) en la que se utiliza una tinción previa de pequeñas porciones de la muestra con anilina azul al 1\%, acidificada con ácido clorhídrico al 1\%. Una vez obtenidos estos, se elaboraron laminillas microscópicas con KARO a concentraciones consecutivas de 30,50 y $80 \%$, las cuales fueron examinadas con ayuda de un microscopio binocular. Para la identificación de las especies se emplearon claves artificiales $(1,2,6)$.

\section{Resultados y discusión}

En el río Manzanares y sus afluentes (incluyendo las zonas inmediatas a la boca y aliviadero del río, con mezcla de aguas marinas) se iden- tificaron 21 especies de fitobentos y macrófitas: 4 Cyanophyta, 9 Chlorophyta, 1 Heterokontophyta, 2 Rhodophyta, 3 Bryophyta y 2 
Magnoliophyta (Familias Hydrocharitaceae y Cymodoceaceae); en la cuencas alta y media se observaron 9 especies y 12 en la cuenca baja (cuadro 1).

La cuenca baja del río Manzanares presentó el mayor número de especies, siendo las más ampliamente distribuidas y frecuentes durante todo el estudio las Chlorophyta Spirogyra majuscula y Cladophora fracta, ambas son abundantes en áreas con eutrofización de alta a moderada, y pueden ser utilizadas como indicadoras de contaminación órganica. Particularmente, C. fracta fue muy abundante en zonas del río cercanas a poblaciones que vierten aguas negras directamente en el cauce, independientemente de la porción de la cuenca estudiada. La abundancia de Cladophora spp. probablemente está relacionada con el incremento de nutrientes orgánicos como el nitrógeno y el fósforo, que redunda en una disminución de la calidad de las aguas. S. majuscula ha sido reportada en sistemas de riego y distribución de aguas contaminadas en Maracay, Estado Aragua, Venezuela (5).

Durante este estudio se confirmó la presencia del alga roja dulceacuícola Thorea riekei en la zona alta de la cuenca del río Manzanares, particularmente en el tributario río Mapurite. Esta alga, que crece en zonas de aguas limpias, frías y de fuerte corriente, fue reportada por primera vez para Venezuela en esta misma área en 1987 (3).

Un elemento importante en la cuenca del río Manzanares es la presencia de musgos acuáticos (Fontinalis spp.) que toleran aguas poco contaminadas con aguas servidas de origen urbano, y actúan como biofiltros que reducen el impacto de este tipo de contaminación, pudiendo utilizarse como biosensores por su capacidad para acumular sustancias tóxicas de origen orgánico y metales pesados. Por otro lado, su abundancia puede constituir un indicativo de la calidad de las aguas, por lo que debe explorarse su potencial en estudios de impacto ambiental.

La distribución irregular de fitobentos y macrófitas, y su abundancia en diferentes porciones de la cuenca del río Manzanares, con presencia de especies indicadoras y/o poco sensibles a la contaminación, independientemente del tramo estudiado, muestran a un cuerpo de agua impactado que puede ser recuperado en un tiempo relativamente corto si se toman las medidas de control pertinentes. 
Cuadro 1. Listado de especies de fitobentos y macrófitas para el río Manzanares, Estado Sucre, Venezuela (A = Aliviadero zona costera).

Especies/Cuencas

Alta Media Baja

Cyanophyta

Nostoc sp.

Phormidium sp.

Polytrotrix sp.

Rivularia sp.

$\mathrm{X}$

$\mathrm{X}$

$\mathrm{X}$

X

Chlorophyta

Spirogyra majuscula Kütz.

Chaetophora sp.

Stygeoclonium fasciculare Kütz.

Chaetomorpha gracilis Kütz.

Cladophora fracta (Müller ex Vahl) Kütz.

Cladophora montagneana Kütz.

Enteromorpha intestinales (L.) Ness

Enteromorpha clahrata (Roth) Greville

Ulva fascista Delile

$\begin{array}{ll}\mathrm{X} & \mathrm{X} \\ & \mathrm{X}\end{array}$

$\mathrm{X}$

$\mathrm{X}$

$\mathrm{X}$

A

$\mathrm{X}$

A

Heterokontophyta

Sargassum vulgare C. Agardh

Rhodophyta

Thorea riekei Bischoff

$\mathrm{X}$

Centroceras clavulatum (C. Agardh) Montagne

Bryophyta

Ricciocarpus sp.

Fontinalis squamosa Hedw.

Fontinalis sp.

$\begin{array}{lll}X & X & X \\ X & X & X \\ X & X & X\end{array}$

Cymodoceaceae

Syringodium filiforme Kützing

Hydrocharitaceae

Thalassia testudinum Banks \& Solander 


\section{Conclusiones}

El río Manzanares presenta una variada vegetación sumergida que incluye algas filamentosas, Bryophyta y Magnoliophyta, resaltando por su abundancia en sitios impactados por contaminación, las Chlorophyta Spirogyra majuscula y Cladophora fracta, particularmente hacia la cuenca media y baja del río.

\section{Agradecimientos}

Los autores expresan su agradecimiento a la Embajada Británica, Consejo de Investigación de la Universidad de Oriente y la Fundación río Manzanares por el financiamiento y la colaboración prestada en el desarrollo del proyecto.

\section{Literatura citada}

1. Cook, C.D.K. 1990. Aquatic Plant Book. SPB Academic Publishing. Netherlands. $228 \mathrm{p}$.

2. Crum, H. 2004. Mosses of the Great lakes forest. Contributions from the University of Michigan Herbarium. 592 p.

3. Ganesan, E.K. y A.J. Lemus. 1987. Note on Venezuela freshwater red algae. Cryptogamie Algologie 8(2): 153-156.

4. Ramírez, M.A. 1995. Recolección y colecciones científicas de macroalgas marinas. p. 417-428. En: K. Alveal, M E. Ferrario, E. C. Oliveira y E. Sar. (Eds). Manual de Métodos Ficológicos. Universidad de Concepción. Chile.

5. Reinefeld, S.H. 1975. Contribución al estudio taxonómico de las algas en los sistemas de distribución de Maracay y sus alrededores. Disponible en la Word Wide Web: http://bibliofcv. veter.ucv.ve/cgi$\mathrm{w}$ i n / b e a l e x . e $\mathrm{x}$ e ? Resumen $=\overline{\mathrm{T}} 040500005970$ / $0 \&$ Nombrebd=BIBLIOFCV

6. Taylor, W.R. 1960. Marine algae of the eastern tropical and subtropical coast of the Americas. Lord Baltimore Press, INC., Universidad de Michigan. 870 p. 